\title{
NIR and Spin Probe Studies on Amylose and Amylopectin
}

\author{
J. J. LINDBERG and TIIA KAILA
}

Department of Wood and Polymer Chemistry, University of Helsinki, Meritullinkatu 1 A, SF-00170Helsinki 17 , Finland

Studies were carried out on the stability of the amylose and amylopectin helix structures in the gel state in the presence of water and dimethylsulfoxide (DMSO) by means of near infrared spectroscopy (NIR) and spin probe(ESR). Observations in the NIR investigation were concentrated on the combination band $(v+\delta)$ of the $\mathrm{O}-\mathrm{H}$ stretching vibration $(v)$ and deformation vibration $(\delta)$ in the $5180-5250 \mathrm{~cm}^{-1}$ region. There were no absorption bands in this region in the NIR spectra of pure DMSO, amylose or amylopectin but amylopectin or amylose swelled with DMSO showed strong absorption at 5200 $\mathrm{cm}^{-1}$. The strength of the hydrogen bond in relation to the stability of the helix structure in different DMSO-water mixtures was calculated with the help of the absorption band data. The helix structure was most stable in $100 \%$ DMSO and 20-30\% DMSO and weakest in $60-70 \%$ DMSO and pure water. The results correlated well with those of ESR experiments in which nitroxyl radicals were used as lables and their ESR spectra as indicators of molecular behaviour in the gels. Distinct maxima and minima were only found in the curves when the sample was allowed to swell for an extended period of time $(4 \mathrm{~h})$ or at high $(343 \mathrm{~K})$ temperatures. Amylopectin needed a longer time or a higher swelling temperature.

Starch is one of the most important adhesives used for technical purposes. Although an immense amount of work has been done on the general adhesion mechanism and gelation phenomena, ${ }^{1}$ very few investigations have been carried out at the molecular level, especially on amylopectin. Erlander and Tobin reported, ${ }^{2}$ for instance, that DMSO and hydrated DMSO stabilize the helix structure of both amylose and amylopectin.

In a recent preliminary report ${ }^{3}$ the remarkable differences in the rheological and hydrogen bonding behaviour of amylose and amylopectin were discussed in terms of limiting viscosity number and relaxation times. This paper is a continuation of the above report and concerns near infrared spectra and spin probe relaxation time data for amylose and amylopectin in the gel state in the presence of water and DMSO.

\section{EXPERIMENTAL}

Technical starch was fractionated into its component amylose and amylopectin by dispersing the granules in water, followed by complex formation of amylose with butanol. ${ }^{3}$ The amylose fraction was purified with ether and the amylopectin fraction with ethanol and ether.

Commercial DMSO, purified by distillation, and distilled water were used. 3-Carboxy-2,2,5,5tetramethylpyrrolidine-1-oxyl was prepared by the method of Rozantsev. ${ }^{4}$

Dissolution tests were carried out at room temperature with different organic solvent mixtures ${ }^{5}$ known to dissolve carbohydrates. DMSO, $\mathrm{H}_{2} \mathrm{O}$ and DMSO $-\mathrm{H}_{2} \mathrm{O}$ mixtures were found to be the best solvents for amylose and amylopectin.

The molecular weight of amylopectin was determined with the light scattering method by dissolving the material in a DMSO - water mixture, the amount of water being $10 \mathrm{~mol} \%$. The weightaverage molecular weight was $17.6 \times 10^{6}$. The molecular weight of amylopectin was $5.2 \times 10^{6}$ when $1 \mathrm{M} \mathrm{KOH}$ was used as solvent.

The amylose fraction used in the investigation was soluble in hot water but insoluble in cold butanolamyl alcohol. ${ }^{3}$ A molecular weight of $0.202 \times 10^{6}$ has been reported for such fractions. ${ }^{6}$

Near infrared spectroscopy and spin probe studies on amylose and amylopectin gels were made in $100 \%$ DMSO, $73 \%$ DMSO $+27 \% \mathrm{H}_{2} \mathrm{O}, 23 \%$ DMSO 
$+\mathrm{H}_{2} \mathrm{O}$ and $100 \% \mathrm{H}_{2} \mathrm{O}$ (mol \%). The study at NIR was made using a Beckman DK 2 spectrometer. Amylose and amylopectin were swelled using a solvent to form a gel. The gel was spread out on the 1 $\mathrm{mm}$ cell and the excess solvent evaporated off using suction at about $343 \mathrm{~K}$. The NIR spectra of the gels were measured over the region 4167 to $14286 \mathrm{~cm}^{-1}$. The spectra of the pure solvents were measured in a 1 $\mathrm{mm}$ cell. The general features of the spin probe method for the characterization of gel systems has been described in several books and journals. ${ }^{7}$

Spin probe studies were made using 3-carboxy2,2,5,5-tetramethylpyrrolidine-1-oxyl radicals as labels. The concentration of radicals in the solvents was $1.8 \mathrm{mg} / \mathrm{ml}$. Two series of experiments were made: In the first one the ESR spectra of the radicals in the amylose and amylopectin gels were measured at room temperature and in the second one at higher temperatures. At room temperature the spectra were measured 1,2,4,6 and $25 \mathrm{~h}$ after preparing the sample. The difference between the stability of the amylose and amylopectin helix structures in different water and DMSO mixtures became apparent after $4 \mathrm{~h}$. Owing to the evaporation of water from the samples the spectra obtained after 6 and $25 \mathrm{~h}$ were omitted. In the second experiment the spectra were measured $1 \mathrm{~h}$ after preparing the sample. The samples were stabilized at each temperature for 5-10 $\mathrm{min}$. The effect of temperature on the stabilization of the helix structure was thus determined. At room temperature a stabilization time of $4 \mathrm{~h}$ was required, but at higher temperature the helix structure stabilized already after $1 \mathrm{~h}$.

The ESR spectra were obtained on a Varian E-4 spectrometer operating at a microwave frequency of $9.086 \mathrm{GHz}$. The microwave power was $1.2 \mathrm{~mW}$. In order to measure the increment in the rotational correlation time of radicals in gel due to the evaporation of solvent, a test was carried out in which the evaporation of solvents for amylose and amylopectin gels were measured in different DMSO - water mixtures by weighing.

\section{RESULTS}

Balevicius, Misiunas and Kimtys $^{8}$ have determined enthalpy and entropy of $\mathrm{H}$-bonding from $\mathrm{OH}_{\text {and }} \mathrm{CH}_{3} \mathrm{NMR}$ chemical shifts and Kleeberg and Luck ${ }^{9}$ have determined the amount and relative $\mathrm{H}$ bond strength of $\mathrm{H}_{2} \mathrm{O}$ molecules which are $\mathrm{H}$ bonded to different polysaccharides by comparison of NIR- and MID-infrared-spectra as well as desorption isotherms. In NIR experiments the characteristic observations were the position and shape of the $\mathrm{H}_{2} \mathrm{O}$ combination band (5300 to 500 $\mathrm{cm}^{-1}$ ) change at relative humidities.

In our experiments, amylose and amylopectin dissolved in DMSO showed strong absorption at $5200 \mathrm{~cm}^{-1}$, which is characteristic of the hydrogen bonds formed between amylopectin (amylose) and DMSO. This is the combination band $(v+\delta)$ of the $\mathrm{O}-\mathrm{H}$ stretching vibration $(v)$ and deformation vibration $(\delta)$. The strength of the hydrogen bond could be calculated by an empirical equation deduced by Badger and Bauer, ${ }^{10}$ eqn. (1), where $f_{1}$

$\Delta H=-k\left(f_{1}-f_{2}\right) / f_{1} \mathrm{kcal} / \mathrm{mol}$

and $f_{2}$ are frequencies of the absorption bands of the free and bonded hydroxyl group respectively, $k$ is an empirical constant and $\Delta H$ is the heat of formation of hydrogen bond.

In this investigation $\Delta H$ values for the hydrogen bonds were calculated by assuming that $f_{2}$ was the sum of the stretching vibration $\left(3652 \mathrm{~cm}^{-1}\right)$ and the deformation vibration $\left(1637 \mathrm{~cm}^{-1}\right)$ of a free water hydroxyl group $\left(=5289 \mathrm{~cm}^{-1}\right)$.

These $\Delta H$ data are not absolute values, but describe the situation in different DMSO - water mixtures formed between amylopectin(amylose) and the solvent. The results are given in Table 1.

In the ESR investigations the rotational correlation times were calculated by the alternative eqns. 2 and 3 , which are based on Kivelson's theory. ${ }^{11}$

Table 1.The combination band of the hydroxyl group in the NIR-region and $\Delta H$ for the hydrogen bonds. $A$ is an empirical constant. Am. $=$ amylose and Ap. $=$ amylopectin.

\begin{tabular}{|c|c|c|c|c|c|}
\hline \multicolumn{2}{|c|}{ Solvent, $\mathrm{mol} \%$} & \multicolumn{2}{|c|}{$v+\delta \mathrm{cm}^{-1}$} & \multicolumn{2}{|c|}{$\Delta H A \times 10^{2} \mathrm{kcal} / \mathrm{mol}$} \\
\hline DMSO & $\mathrm{H}_{2} \mathrm{O}$ & Am. & Ap. & Am. & Ap. \\
\hline 100 & 0 & 5189 & 5202 & 1.9 & 1.7 \\
\hline 73 & 27 & 5197 & 5216 & 1.7 & 1.4 \\
\hline 23 & 77 & 5187 & 5196 & 1.9 & 1.8 \\
\hline 0 & 100 & 5216 & 5243 & 1.4 & 0.9 \\
\hline
\end{tabular}


$\tau_{1}=8.7 W_{0}\left[\left(h_{0} / h_{1}\right)^{1 / 2}-\left(h_{0} / h_{-1}\right)^{1 / 2}\right] \times 10^{-10} \mathrm{~s}$

$\tau_{2}=7.9 W_{0}\left[\left(h_{0} / h_{1}\right)^{1 / 2}+\left(h_{0} / h_{-1}\right)^{1 / 2}-2\right] \times 10^{-10} \mathrm{~s}$

$W_{0}$ is the line width of the centre line in the ESR spectrum and $h_{1}, h_{0}$ and $h_{-1}$ are the intensities of the low, center and high field components. In general, mean values of $\tau_{1}$ and $\tau_{2}$ are used.

We have previously investigated the rotational correlation times of the nitroxyl radicals in DMSO - water mixtures as a function of the molar composition of the DMSO. ${ }^{12}$ Since $\tau$ was not a constant at different solvent composition the correlation times of radicals in DMSO - water mixtures were subtracted in the present investigation from the corresponding values of $\tau$ for the radicals in the gels. It was also found in the same investigation that the rotational correlation times for constant solvent composition were nearly constant over the temperature range $293-335 \mathrm{~K}$ in both water and in DMSO. Thus the correlation times describe only the behaviour of amylose and amylopectin.

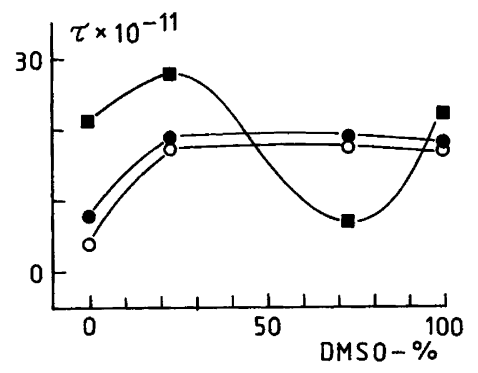

Fig. 1. Spin probe investigation at RT on amylopectin swelled in DMSO - water mixtures. Swelling times: $\mathrm{O}, 1 \mathrm{~h}$;, $2 \mathrm{~h} ; \square, 4 \mathrm{~h}$.

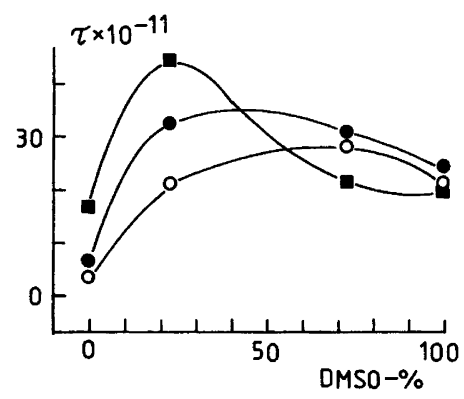

Fig. 2. Spin probe investigation at RT on amylose swelled in DMSO - water mixtures. Swelling times: $\mathrm{O}, 1 \mathrm{~h} ; \mathrm{O}, 2 \mathrm{~h} ; \square, 4 \mathrm{~h}$.

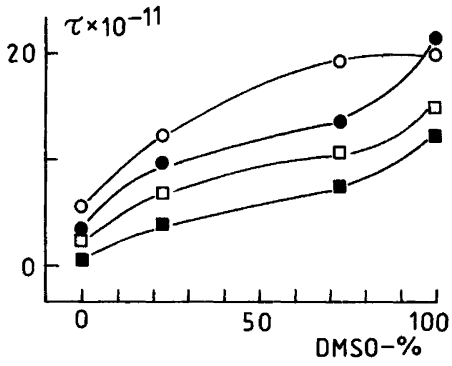

Fig. 3. Spin probe investigation at various temperatures on amylopectin swelled for $1 \mathrm{~h}$ in DMSO - water mixtures. Temperatures: $\bigcirc, 294 \mathrm{~K}$; , $313 \mathrm{~K} ; \square, 325 \mathrm{~K}$; $\square, 335 \mathrm{~K}$.

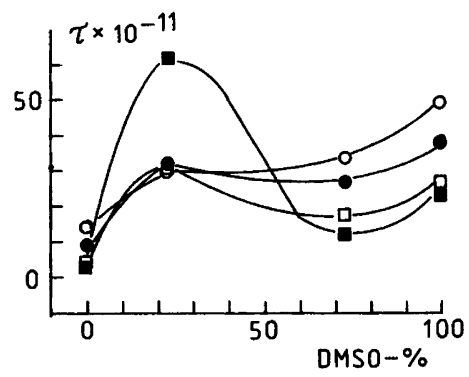

Fig. 4. Spin probe investigation at various temperatures on amylose swelled for $1 \mathrm{~h}$ in DMSO -water mixtures. Temperatures: $\bigcirc, 306 \mathrm{~K}$;, 313 $\mathrm{K} ; \square, 325 \mathrm{~K} ; \square, 335 \mathrm{~K}$.

Figs. 1 and 2 show a graph of the rotational correlation time of nitroxyl radicals in DMSO - water-amylopectin and amylose gels against different DMSO - water molar compositions when the sample has been swollen for 1,2 and $4 \mathrm{~h}$. It can be seen from the curves that after $4 \mathrm{~h}$ of gelation clear maximum and minimum relaxation times are observed both in amylopectin and amylose gels. The correlation times of radicals in DMSO - wateramylopectin and amylose gels vs. DMSO molar composition in different temperatures are shown in Figs. 3 and 4. The amylose curves have a strong maximum and minimum at higher temperatures. There is also a minimum and maximum in the amylopectin curves but they are much weaker.

It was found in the investigation of solvent evaporation from amylose and amylopectin gels that amylopectin binds DMSO-water mixtures considerably better that amylose does: After $20 \mathrm{~h}$ of gelation $20 \%$ solvent (water) had evaporated from 
the amylopectin - DMSO - water gel and $40 \%$ from the amylose - DMSO - water gel. $75 \%$ of the water evaporated from both gels after $20 \mathrm{~h}$ storage, when no DMSO was present.

\section{DISCUSSION}

A polar molecule affects the surrounding molecules; the electric field associated with a dipole causes slight displacement of the electrons and nuclei of surrounding molecules. The DMSO-molecule $\left({ }^{-} \mathrm{O}-{ }^{+} \mathrm{S}\left(\mathrm{CH}_{3}\right)_{2} \text {, dipole moment } 4.3 \mathrm{D}\right)^{13}$ has a strong attraction for the hydroxyl group of amylose and amylopectin, and therefore would induce a greater polarity ${ }^{2}$ in it. This greater polarity would in turn produce a stronger attraction for the neighbouring hydroxyl group on the adjacent glucose unit. Hence, DMSO would strengthen the well-known amylose and amylopectin helix structures. The same type of complexation could occur in aqueous solutions between hydroxyl groups and water molecules (dipole moment $1.87 \mathrm{D}$ ). ${ }^{14}$

Since DMSO has a greater dipole moment than water, it would strengthen the hydrogen bond between the adjacent glucose units more than water. It can be seen from Table 1 that in the NIRexperiments, the hydrogen bonds of DMSOamylopectin gel are twice as strong as those of the water-amylopectin gel. This is in good agreement with the difference between the dipole moments of DMSO and water molecules. It is evident that amylose has stronger hydrogen bonds than amylopectin in both solvents.

The ESR investigation also showed that DMSO stabilizes the helix structure better than water, and that amylose has a more rigid structure than amylopectin. Since the evaporation of water from gels further increased the correlation time, especially in $100 \%$ water, the numerical difference between the hydrogen bond in DMSO - amylopectin gels and water - amylopectin gels could not be determined on the basis of the ESR experiments.

As only two mixtures of DMSO - water were used in the investigation, the positions of maximum and minimum in the curves (Figs. 1-4) are not quite exact. However, in both the NIR and ESR investigations the helix structure was most stable in $20-30 \%$ DMSO and least stable in $60-70 \%$ DMSO. In $60-70 \%$ solutions the hydrated DMSO forms a hydrogen bond with amylopectin and amylose. As noted above, the DMSO forms an A- region. Hence the water molecule hydrated to this molecule will be more polar than normal water molecules. Consequently, the hydrated DMSO will form a stronger hydrogen bond with the amylopectin than normal water does, but weaker than that with DMSO (Table 1).

The stabilization of the helix structure in $20-30 \%$ DMSO could be explained by the fact that DMSO is hydrated in water with two water molecules. ${ }^{15,16}$ Both of these molecules would be able to form hydrogen bonds with the hydroxyl between the chains and the gel would bestabilized. This could also explain the great increase in stabilization.

Amylopectin needed a longer swelling time or higher temperature to form a complex with DMSO (or with water) than amylose. The distinct maximum and minimum in the amylopectin curves were only found in the NIR investigation when the sample was treated at $343 \mathrm{~K}$ and in the ESR experiments when the gels were swollen for $4 \mathrm{~h}$. This is due to the structural difference between amylose and amylopectin. Owing to the extensive branching in amylopectin, it is difficult for the solvent to penetrate into the inner region of the amylopectin molecule. In addition to this, amylopectin already has a rigid structure and the DMSO $2 \mathrm{H}_{2} \mathrm{O}$ bridges could not stabilize the amylopectin gel as they did in the unbranched amylose gel. The same effect has also been observed in other non-linear macromolecules e.g. lignin, ${ }^{17}$ where a penetration of the solvent, pyridine, into the inner region of the lignin network was only observed at elevated temperatures.

The above results indicate that hydrogen bond formations in linear helix and non-linear structures possessing helical areas are very variable owing to differences in penetration ability. Furthermore, the association effects are strongly enhanced following chain branching. This effect has earlier been considerably underestimated when considering the association and solubility phenomena in polymeric systems.

\section{REFERENCES}

1. Wolfrom, M. L. and Khadem, H. E. In Whistler, R. L. and Paschall, E. F., Eds., Starch: Chemistry and Technology, Academic, New York 1965.

2. Erlander, S. R. and Tobin, R. Die Makromolekulare Chemie III (1968) 194.

3. Penttinen, E.-L. Diploma work, Helsinki 1977; Wartiowaara-Tuompo, J., Lindberg, J. J. and Penttinen, E.-L. Kem. Kemi 4 (1977) 554.

Acta Chem. Scand. B 34 (1980) No. 10 
4. Rozantsev, E. G. Free Nitroxyl Radicals, Plenum, New York 1970.

5. Okajima, K. Chem. Scr. (1978) 102.

6. Banks, W., Greenwood, C. T. and Sloss, J. Carbohydr. Res. 11 (1969) 399.

7. Törmälä, P. and Lindberg, J. J. In Ivin, K. J., Ed., Structural Studies of Macromolecules by Spectroscopic Methods, Wiley, London 1976, Chapter 14.

8. Balevicius, V., Misiunas, G. and Kimtys, L. Liet. Fiz. Rinkinys 6 (1977) 749.

9. Kleeberg, H. and Luck, W. A. P. The Hydration of Polysaccharides: Comparison of Infrared Spectra and Sorption Isotherms, 27th IUPAC Congress, Helsinki 1979.

10. Badger, R. M. and Bauer, S. H. J. Chem. Phys. 5 (1937) 839.

11. Kivelson, D. J. Chem. Phys. 33 (1960) 1094.

12. Bulla, I., Törmälä, P. and Lindberg, J. J. Acta Chem. Scand. A 29 (1975) 89.

13. Martin, D. and Hauthal, H. G. Dimethylsulfoxid, Akademieverlag, Berlin 1971.

14. Handbook of Chemistry and Physics, 58th Ed., $1977-1978$.

15. Cowie, J. M. C. and Toporowski, P. M. Can. J. Chem. 39 (1961) 2240.

16. Wu,N.M. and Malinin, T.I. Anal.Chem.52(1980) 186; Tommila, E. and Murto, M.-L. Acta Chem. Scand. 17 (1963) 1947.

17. Törmälä,P.,Lindberg,J.J. and Lehtinen, S. Paper and Timber (Finland) 57 (1975) 601.

Received June 23, 1980. 